\title{
A case report of mesenteric mucinous cystoadenoma with review of the literature
}

\author{
Alessandro Del Gobbo ${ }^{\dagger}$, Manuela Bimbatti ${ }^{\dagger}$, Stefano Ferrero ${ }^{*}$
}

\begin{abstract}
Background: Few cases of primary retroperitoneal mucinous cystoadenoma, a rare benign tumor, have been reported in the literature so far. The pathogenesis of this tumour is not completely understood yet.

Our case is particularly significant since the localization in the mesentery has been described only once before in the literature. Unless biologically benign, this tumour can cause relevant clinical symptoms related to the size and site (compression or obstruction of organs).

Case presentation: We describe the case of a 52-years old woman who had presented with abdominal pain and underwent surgery in order to remove a palpable lump in the mesentery with histological diagnosis of primary mucinous cystoadenoma. The patient was followed-up for two years with no evidence of recurrence.

Conclusions: Mucinous cystoadenoma is more frequent in women, particularly when there is history of one or more pregnancies. A complete preoperative study with abdominal and pelvic tomographic images and an accurate physical examination are essentials for the management of the patient. Surgical resection is the only way to treat mucinous cystoadenomas, and to have the histological confirmation that the removed mass is a benign tumor.
\end{abstract}

\section{Background}

A few cases of retroperitoneal mucinous cystoadenoma (RMC), a rare benign tumor, are reported in the literature [1-6]. This is the second case reported in the literature of mesenteric localization of mucinous cystoadenoma to our best knowledge [1].

Cystoadenoma causes symptoms linked to its size and site as do many masses localized in the abdominal cavity or retroperitoneum, where frequently the adjacent structures like blood vessels, ureters, small and/or large bowel tracts can be compressed or obstructed. The symptom more frequently described in these cases is the abdominal pain, sometimes exacerbated by the compression practiced on the mass during medical examination.

\section{Case Presentation}

A 52 year-old woman came to Hospital complaining a vague feeling of heaviness in right lower quadrant in the

\footnotetext{
* Correspondence: stefano.ferrero@unimi.it

+ Contributed equally

Department of Medicine, Surgery and Dentistry, Division of Pathology, AO S. Paolo e Fondazione IRCCS Ospedale Maggiore Policlinico, Mangiagalli and Regina Elena, University of Milan Medical School, Milan, Italy
}

last few months. The woman had had two pregnancies and the anamnesis did not put into evidence any pathologically relevant note.

The medical examination revealed a deep palpable mass in the abdomen; blood tests were normal, except for the modest raising of the ALT equal to $65 \mathrm{U} / \mathrm{Ls}$ (normal range 7-41), CEA equal to 13.58 (normal range: 0.0-4.6), and of the CA 19.9 equal to 43.55 (normal range 0.0-37).

Transvaginal ultrasound features of the mass showed an egg-shaped cystic mass of nearly $14 \mathrm{~cm}$ in diameter, uniloculated, with liquid content, and thickened walls with foci of calcification. Colonoscopy revealed compression of terminal ileum and right colon with normal mucosa. The lump was deeply located in the right lower quadrant, in anatomical relationship with ileum, and causing the compression of the right ureter; the ovaries and the female reproductive system were normal. The patient did not undergo abdominal or pelvic computed tomography, since both of them were considered to be unnecessary by the surgeon.

The surgical intervention allowed the removal of the retroperitoneal mass with terminal ileum, right colon, 


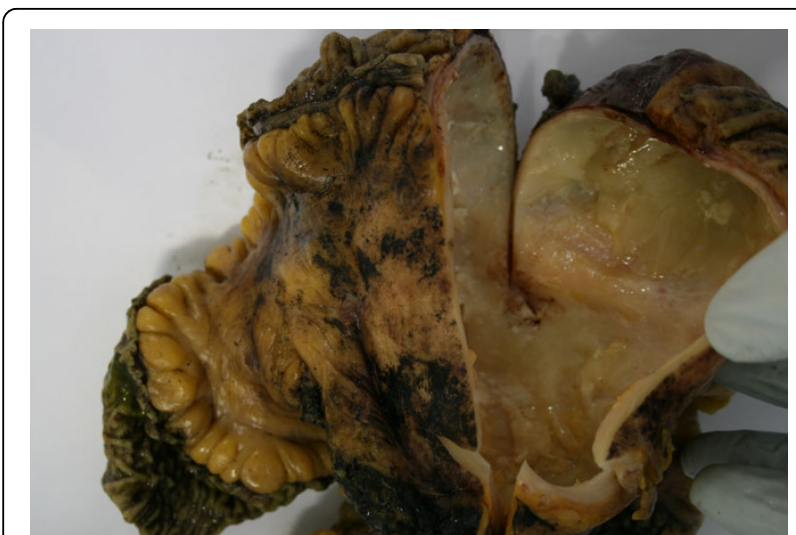

Figure 1 Gross view of the specimen

right ovarian vessels and right ureter, which were embedded in the mass. The continuity of the right ureter was guaranteed with a "double J" prosthesis uretero-ureteroanastomosis. Intraoperative examination excluded neoplastic involvement of the resected right ureter.

A mass of $10,5 \mathrm{~cm}$ of major diameter was entirely embedded in the mesentery, from which it was easily removed except for an area of firm adhesion to surrounding connective tissue corresponding to terminal ileum, the external surface was smooth and the cut-up showed a cystic lesion with smooth internal surface, containing mucinous whitish fluid (fig. 1).

The wall and the mucosa of both small and large bowel and the right ureter appeared grossly uninvolved by the lesion.

The most common differential diagnoses include other neoplastic lesions such as cystic teratoma, Mullerian cysts or epidermoid cysts, cystic change in solid

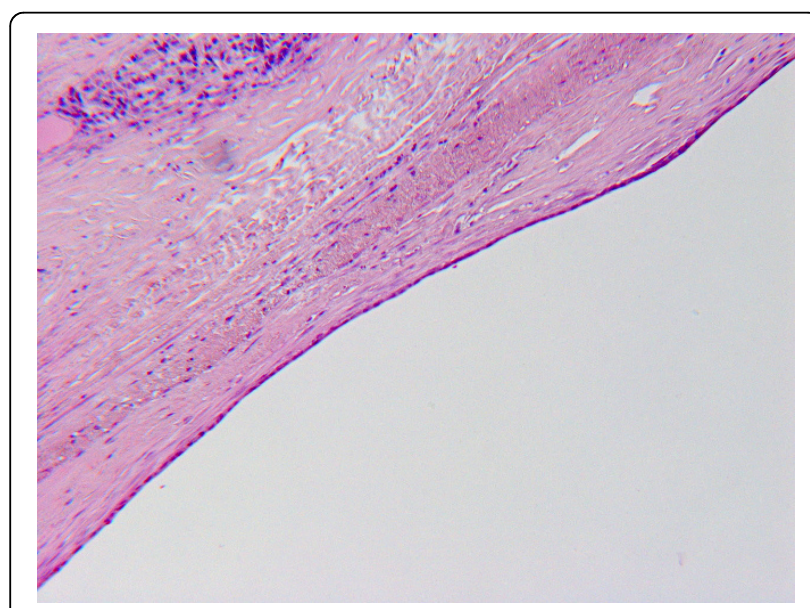

Figure $2 \mathrm{~A}$ the microscopic view, it can be seen the cuboidal epithelium, sometimes cylindrical, with mucus-secreting cells (HE, 10X).

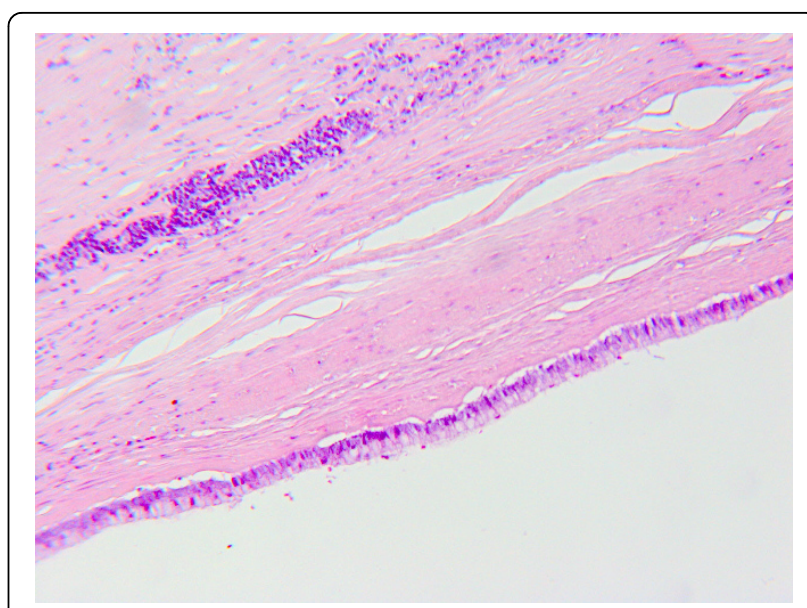

Figure 3 Another microscopic view of the epithelium (HE, 20x).

neoplasms as in paraganglioma, neurogenic tumor or pseudomixoma retroperitonei and some non-neoplastic lesions such as pancreatic pseudocysts, lymphocele, urinoma and hematoma.

Under histological examination the cyst wall was composed of a tick layer of connective fibrous tissue covered by a single-layer of cuboidal cells with interspersed typical goblet cells (fig. 2 and 3); ovarian-like mesenchymal stroma was not seen. We performed Alcian blue staining in order to highlight the mucinous secretion of the surface epithelial cells (fig. 4).

Immunoistochemistry included cytokeratins 7 (DAKO; clone OV-TL 12/30; 1:30) and 20 (DAKO; clone Ks20.8, 1:50), which both identified the lining epithelium. Calretinin (DAKO; clone DAK-Calret 1, 1:50), a marker for mesothelial cells lineage was negative as well as vimentin (DAKO; clone V9, 1:50), (fig. 5a-d).

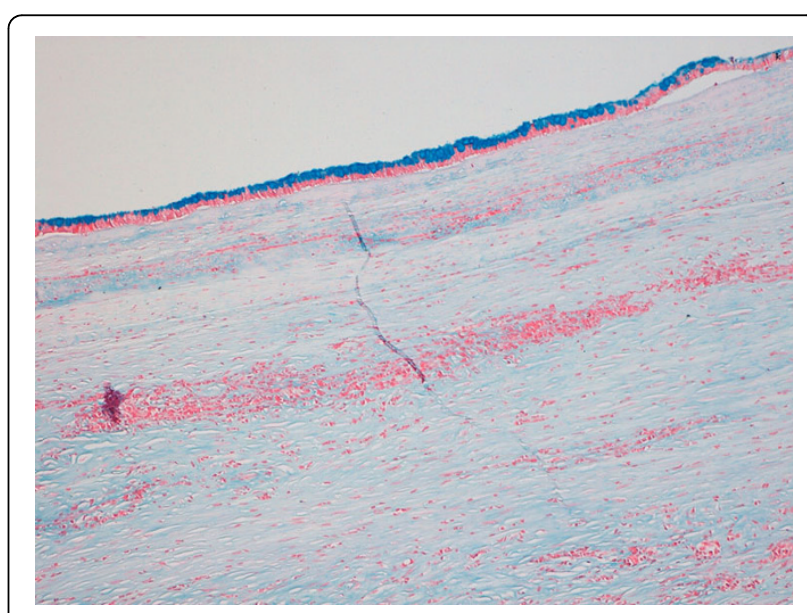

Figure 4 Alcian blue staining identifies the mucinous secretion of the surface epithelium (10x). 


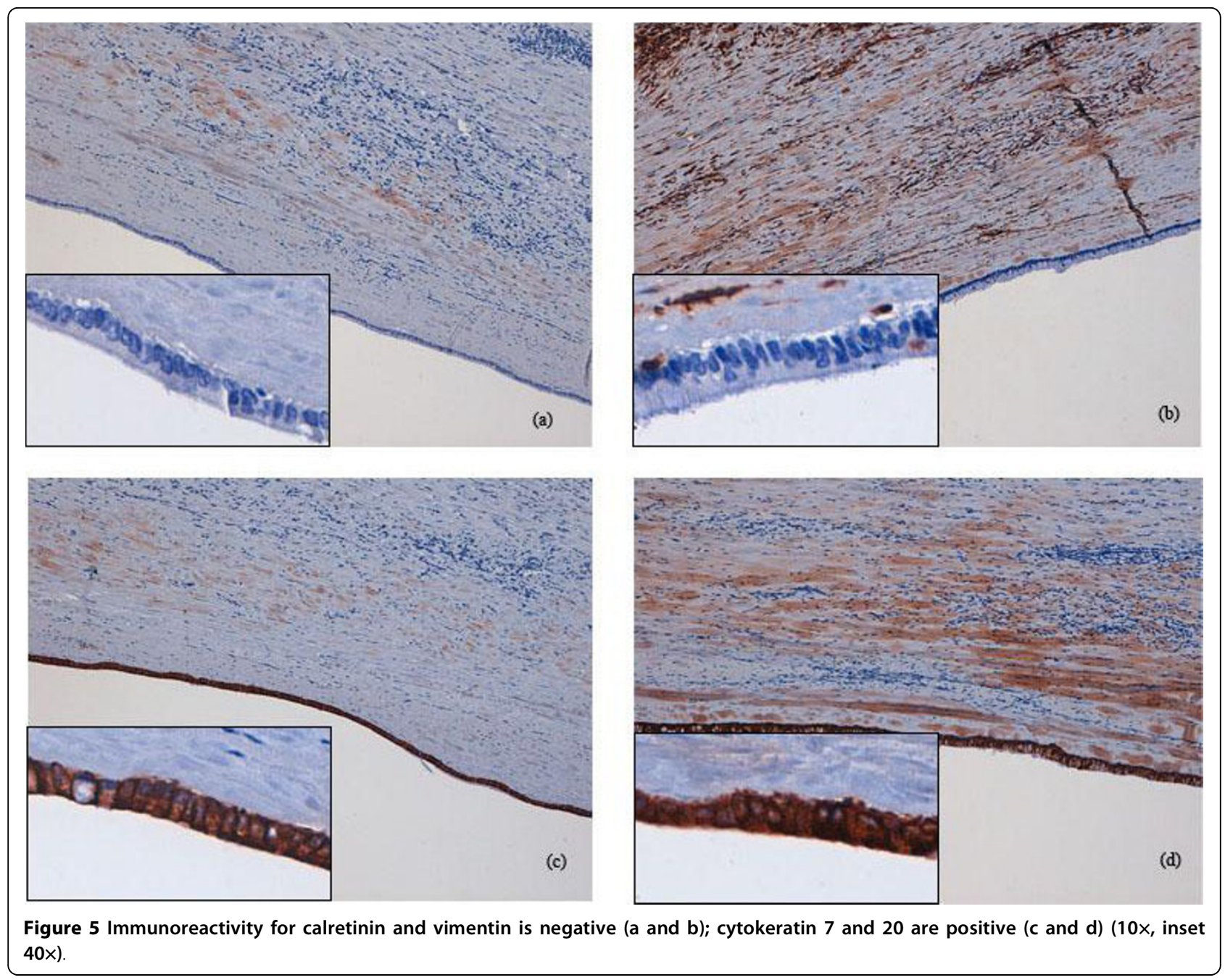

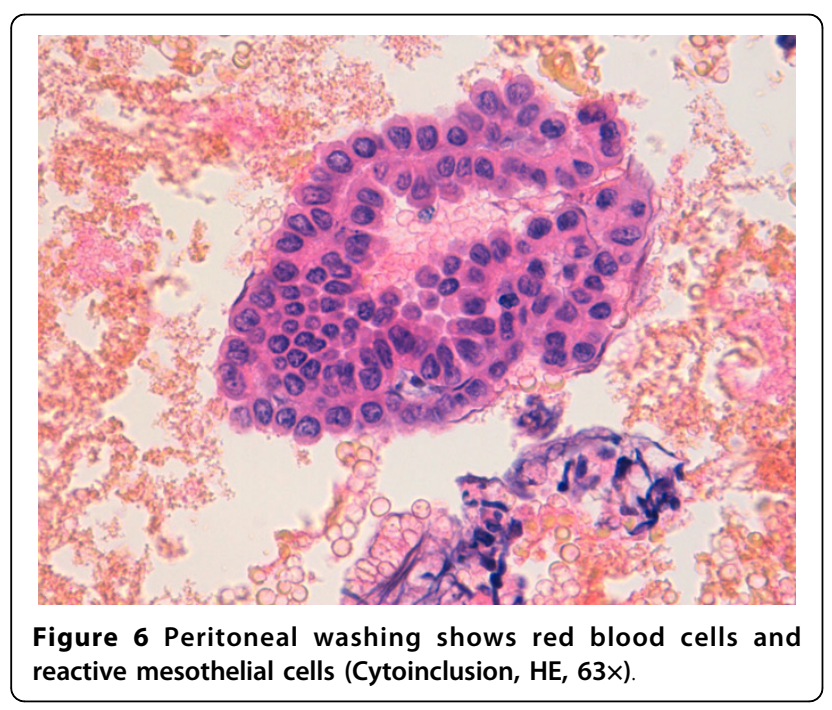

Because of the morphological and immunoistochemical features we made diagnosis of mesenteric mucinous cystoadenoma.

The cytological examination of peritoneal washing fluid showed reactive mesothelial cells, and it resulted negative for the presence of malignant tumor cells. (fig. 6).

The patient was discharged after ten days, without complications. Two years after surgery, the patient is still in good health.

There are two theories about the genesis of retroperitoneal mucinous cystoadenoma: for its similarity with the ovarian cystoadenoma, it can originate from ectopic ovarian tissue [6].

The second hypothesis sustains that these cysts derive from the invagination of pluripotent embrionary epithelium and subsequent mucinous metaplasia, which would 
give origin to a mucinous cyst, and later, to the mucinous cystoadenoma (coelomatic hypotesis) [2]. According to this hypothesis, during the $4^{\circ}$ week of pregnancy the embryo runs into complex processes of folding that will bring it to its three-dimensional form, with the constitution of the body cavities, organs and apparatus. We suppose that during these processes some cells (that will constitute the ovarian epithelium) can migrate in the serous cavity that will become the peritoneal one and, if adequately stimulated, these cells could undergo into mucinous metaplasia, with characteristics of benignity.

This case is similar to the one reported by Chen et al [1] regarding the age of the patient and symptoms, but it differs for the histology (the previous case has borderline malignancy) and for the surgical management (in the first case, surgeons voided the cyst under ultrasonic guidance before removing the mass).

\section{Conclusions}

This case is relevant for the site of occurrence: most cystoadenomas are located in the ovaries, and mesenteric localization is rare.

Also reported symptoms are peculiar: the patient did not complain of pain or other symptoms related to compression of the right ureter (which was embedded in the mass with the right ovarian blood vessels) or bowel, but just a vague feeling of abdominal heaviness.

In our view, preoperative abdominal/pelvic computed tomography would have been useful

\footnotetext{
Acknowledgements

Written informed consent was obtained from the patient for publication of this case report and any accompanying images. A copy of the written consent is available for review by the Editor-in-Chief of this journal.
}

\section{Authors' contributions}

All authors read approved the manuscript. ADG and MB analysed, interpreted the patient's data and drafted the manuscript. SF revised the pathology data and supervised the case report.

All authors read and approved the final manuscript.

\section{Competing interests}

The authors declare that they have no competing interests.

Received: 21 April 2010 Accepted: 14 September 2010

Published: 14 September 2010

\section{References}

1. Chen JS, Lee WJ, Chang YJ, Wu MZ, Chiu KM: Laparoscopic resection of a primary retroperitoneal mucinous cystoadenoma: report a case. Surg Today Jpn J Surg 1998, 28:343-345.

2. Isse K, Harada K, Suzuki Y, Ishiguro K, Sasaki M, Kajiura S, Nakanuma Y: Retroperitoneal mucinous cystoadenoma: report of two cases and review of literature. Pathology International 2004, 54:132-138.

3. Kehagias DT, Karvounis EE, Fotopoulos A, Gouliamos AD: Retroperitoneal mucinous cystoadenoma. Europ J of Obst \& Gynnec and Reproduct Biol 1999, 82:213-215.

4. Sheen-Chen SM, Eng HL: Retroperitoneal mucinous cystoadenoma. Dig Diseas and Science 2006, 51:752-753.
5. Yan SL, Lin H, Kuo CL, Wu HS, Huang MH, Lee YT: Primary retroperitoneal mucinous cystoadenoma: report of a case and review of literature. Word J Gastroenterol 2008, 37:5769-5772.

6. Yang DM, Jung DH, Kim H, Kang JH, Kim SH, Kim JH, Hwang HY: Retroperitoneal cystic masses; $\mathrm{CT}$, clinical an pathologic findings and literature review. Radiographics 2004, 24:1353-1365.

\section{Pre-publication history}

The pre-publication history for this paper can be accessed here: http://www.biomedcentral.com/1471-230X/10/105/prepub

doi:10.1186/1471-230X-10-105

Cite this article as: Del Gobbo et al:: A case report of mesenteric mucinous cystoadenoma with review of the literature. BMC

Gastroenterology 2010 10:105.

\section{Submit your next manuscript to BioMed Central and take full advantage of:}

- Convenient online submission

- Thorough peer review

- No space constraints or color figure charges

- Immediate publication on acceptance

- Inclusion in PubMed, CAS, Scopus and Google Scholar

- Research which is freely available for redistribution

Submit your manuscript at www.biomedcentral.com/submit 